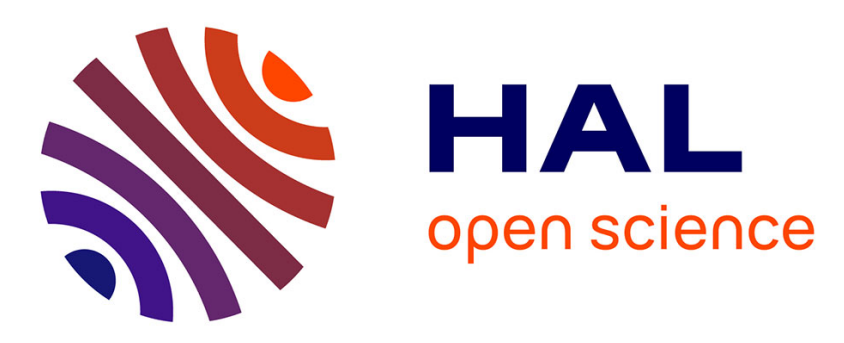

\title{
Perturbation Finite Element Method for Magnetic Model Refinement of Air Gaps and Leakage Fluxes
}

Patrick Dular, Ruth V. Sabariego, Mauricio Valencia Ferreira da Luz, Patrick Kuo-Peng, Laurent Krähenbühl

\section{- To cite this version:}

Patrick Dular, Ruth V. Sabariego, Mauricio Valencia Ferreira da Luz, Patrick Kuo-Peng, Laurent Krähenbühl. Perturbation Finite Element Method for Magnetic Model Refinement of Air Gaps and Leakage Fluxes. IEEE Transactions on Magnetics, 2009, 45 (3), pp.1400-1403. 10.1109/TMAG.2009.2012643 . hal-00364759

\section{HAL Id: hal-00364759 \\ https://hal.science/hal-00364759}

Submitted on 27 Feb 2009

HAL is a multi-disciplinary open access archive for the deposit and dissemination of scientific research documents, whether they are published or not. The documents may come from teaching and research institutions in France or abroad, or from public or private research centers.
L'archive ouverte pluridisciplinaire HAL, est destinée au dépôt et à la diffusion de documents scientifiques de niveau recherche, publiés ou non, émanant des établissements d'enseignement et de recherche français ou étrangers, des laboratoires publics ou privés. 


\title{
Perturbation Finite Element Method for Magnetic Model Refinement of Air Gaps and Leakage Fluxes
}

\author{
Patrick Dular $^{1,2}$, Ruth V. Sabariego ${ }^{1}$, Mauricio V. Ferreira da Luz ${ }^{3}$, Patrick Kuo-Peng ${ }^{3}$, and Laurent Krähenbühl ${ }^{4}$ \\ ${ }^{1}$ Department of Electrical Engineering and Computer Science, ACE, University of Liège, B-4000 Liège, Belgium \\ ${ }^{2}$ FNRS, University of Liège, B-4000 Liège, Belgium \\ ${ }^{3}$ GRUCAD/EEL/UFSC, 88040-970 Florianópolis, Santa Catarina, Brazil \\ ${ }^{4}$ Université de Lyon, Ampère (UMR CNRS 5005), École Centrale de Lyon, F-69134 Écully Cedex, France
}

\begin{abstract}
Model refinements of magnetic circuits are performed via a subproblem finite element method based on a perturbation technique. An approximate problem considering ideal flux tubes and simplified air-gap models is first solved. It gives the sources for a finite element perturbation problem considering the actual air gaps and flux tubes geometries with the exterior regions. The procedure simplifies both meshing and solving processes, and allows to quantify the gain given by each model refinement.
\end{abstract}

Index Terms-Finite-element method (FEM), magnetic circuits, perturbation method.

\section{INTRODUCTION}

$\mathbf{T}$ HE perturbation of finite element (FE) solutions provides clear advantages in repetitive analyses [1] and helps improving the solution accuracy [2]. It allows to benefit from previous computations instead of starting a new complete FE solution for any variation of geometrical or physical data. It also allows different problem-adapted meshes and computational efficiency due to the reduced size of each subproblem.

A perturbation FE method is herein developed for refining the magnetic flux distribution in magnetic circuits starting from simplified FE models, which are based on both ideal flux tubes [3] and thin-shell air-gap models [4]. The developments are performed for the magnetic vector potential FE magnetostatic formulation, paying special attention to the proper discretization of the constraints involved in each subproblems. The method is validated on test problems.

\section{A SERIES OF COUPLED SUbPRoblems}

\section{A. Canonical Magnetostatic Problem in a Strong Form}

A canonical magnetostatic problem $p$ is defined in a domain $\Omega_{p}$, with boundary $\partial \Omega_{p}=\Gamma_{p}=\Gamma_{h, p} \cup \Gamma_{b, p}$ (possibly at infinity), of the 2-D or 3-D Euclidean space. Subscript $p$ refers to the associated problem $p$.

The equations, material relations, boundary conditions (BCs) and interface conditions (ICs) of problem $p$ are

$$
\begin{gathered}
\operatorname{curl} \boldsymbol{h}_{p}=\boldsymbol{j}_{p}, \quad \operatorname{div} \mathbf{b}_{p}=0, \quad \boldsymbol{b}_{p}=\mu_{p} \boldsymbol{h}_{p}+\boldsymbol{b}_{s, p}, \\
\boldsymbol{n} \times\left.\boldsymbol{h}_{p}\right|_{\Gamma_{h, p}}=0,\left.\quad \boldsymbol{n} \cdot \boldsymbol{b}_{p}\right|_{\Gamma_{b, p}}=0, \\
{\left[\boldsymbol{n} \times \boldsymbol{h}_{p}\right]_{\gamma_{p}}=\boldsymbol{j}_{s u, p}, \quad\left[\boldsymbol{n} \cdot \boldsymbol{b}_{p}\right]_{\gamma_{p}}=\boldsymbol{b}_{s u, p},}
\end{gathered}
$$

where $\boldsymbol{h}_{p}$ is the magnetic field, $\boldsymbol{b}_{p}$ is the magnetic flux density, $\boldsymbol{j}_{p}$ is limited to the source electric current density $\boldsymbol{j}_{s, p}, \mu_{p}$ is the magnetic permeability and $\boldsymbol{n}$ is the unit normal exterior to $\Omega_{p}$.

Manuscript received October 07, 2008. Current version published February 19, 2009. Corresponding author: P. Dular (e-mail: Patrick.Dular@ulg.ac.be).

Color versions of one or more of the figures in this paper are available online at http://ieeexplore.iee.org.

Digital Object Identifier 10.1109/TMAG.2009.2012643
The field $\boldsymbol{b}_{s, p}$ is a possible volume source. It is usually used for fixing a remnant induction.

The notation $[\cdot]_{\gamma}=\left.\cdot\right|_{\gamma^{+}}-\left.\cdot\right|_{\gamma^{-}}$expresses the discontinuity of a quantity through any interface $\gamma\left(\right.$ with sides $\gamma^{+}$and $\gamma^{-}$) in $\Omega_{p}$, which is allowed to be non-zero. The associated surface fields $\boldsymbol{j}_{s u, p}$ and $\boldsymbol{b}_{s u, p}$ are usually zero, defining classical essential or natural ICs for the physical fields. Nonzero quantities define possible surface sources.

A key element of the developed method is to define the volume and surface sources of problem $p$ from parts of solutions of other problems.

\section{B. Each Subproblem Defines a Perturbation}

The objective is solving successive problems, the addition of which being the solution of a complete problem. For an ordered set $P$ of problems, the complete solution is

$$
\boldsymbol{u}=\sum_{p \in P} \boldsymbol{u}_{p}, \text { with } \boldsymbol{u} \equiv \boldsymbol{h}, \boldsymbol{b}, \boldsymbol{j}_{s u}, \boldsymbol{b}_{s u}, \ldots
$$

At the discrete level, each problem is defined in its own domain and mesh, which decreases the problem complexity and allows distinct mesh refinements.

Also, such a superposition of solutions allows each subproblem to satisfy some constraints and relations that are not shared with the complete problem. Consequently, each subproblem is generally perturbed by all the others and each solution has to be then calculated as a series of corrections, i.e.,

$$
\boldsymbol{u}_{p}=\sum_{i} \boldsymbol{u}_{p, i}=\boldsymbol{u}_{p, 1}+\boldsymbol{u}_{p, 2}+\ldots
$$

The calculation of the correction $\mathbf{u}_{p, i}$ in a problem $p, i$ is kept on till convergence up to a desired accuracy. Each correction must account for the influence of all the previous corrections $\mathbf{u}_{p, j}$ of the other subproblems, with $j$ the last iteration index for which a correction is known. Initial solutions $\mathbf{u}_{p, 0}$ are set to zero. The iterative process is justified by the fact that a correction can become a significant source for any of its source problems, which is proper to large perturbation problems. In addition to the iterations between subproblems, classical inter-problem iterations are needed in nonlinear analyses. The global quantities linearly related to each correction (fluxes and magnetomotive forces [3]) are to be added to give their complete values. 

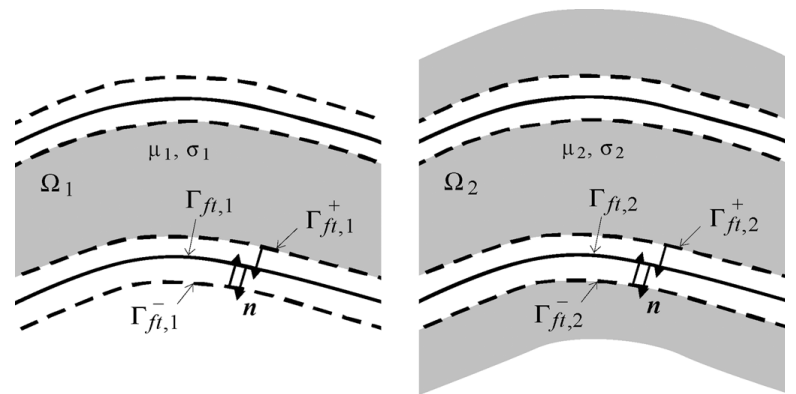

Fig. 1. Domains for the ideal $(p=1$, left) and real $(p=2$, right) flux tube problems.

\section{Volume and Surface Perturbations}

A change of BCs or ICs from problem $q$ to $p$ is defined via surface sources (or interface-type sources) fixing the possible trace discontinuities of $\boldsymbol{h}_{p}$ and $\boldsymbol{b}_{p}$ in terms of the solution of problem $q$.

A change of a material property in a volume region defines a volume source (or a region-type source) in the associated material relation. For a change of permeability, from $\mu_{q}$ for problem $q$ to $\mu_{p}$ for problem $p$, the volume source in the $\boldsymbol{b}-h$ relation (1c) is of the form

$$
\boldsymbol{b}_{s, p}=\left(\mu_{p}-\mu_{q}\right) h_{q}
$$

This way, summing both relations $\boldsymbol{b}_{q}=\mu_{q} \boldsymbol{h}_{q}$ (with e.g. $\boldsymbol{b}_{s, q}=$ 0 ) and $\boldsymbol{b}_{p}=\mu_{p} h_{p}+\boldsymbol{b}_{s, p}$ gives the relation that is valid for the superposition of solutions $q$ and $p$, i.e. $\boldsymbol{b}_{q}+\boldsymbol{b}_{p}=\mu_{q} \boldsymbol{h}_{q}+\mu_{p} \boldsymbol{h}_{p}+$ $\left(\mu_{p}-\mu_{q}\right) \boldsymbol{h}_{q}=\mu_{p}\left(\boldsymbol{h}_{q}+\boldsymbol{h}_{p}\right)$. In the same way, the $\boldsymbol{h}-\boldsymbol{b}$ relation would be

$$
\boldsymbol{h}_{p}=\mu_{p}^{-1} \boldsymbol{b}_{p}+\boldsymbol{h}_{s, p}, \text { with } \boldsymbol{h}_{s, p}=\left(\mu_{p}^{-1}-\mu_{q}^{-1}\right) \boldsymbol{b}_{q} .
$$
give

A generalization of (4) to any number of subproblems would

$$
\boldsymbol{b}_{s, p}=\left(\mu_{p}-\mu_{q}\right) \sum_{r \in P, r \neq p} \boldsymbol{h}_{r}
$$

with $q$ the last solved problem.

Note that the differential (1a) and (1b) remain unchanged for each subproblem, their addition directly giving the associated equation governing the complete solution.

\section{Perturbation Problems}

\section{A. From Ideal to Real Flux Tubes}

In a first problem $p=1$, the magnetic flux is forced to flow only in a subregion with perfect flux walls, i.e. a set of flux tubes $\Omega_{1}=\Omega_{f t, 1}$ of the whole domain $\Omega$ (of the complete problem). A second problem $p=2$ considers then the flux walls become permeable. This allows leakage flux in the exterior region $\Omega \backslash \Omega_{1}$ and leads to a change of the flux distribution in $\Omega_{1}$. A solution refinement is thus obtained.

In problem 1, the ideal flux tubes are considered with a BC of zero normal magnetic flux density on their boundaries $\Gamma_{f t, 1}=$ $\partial \Omega_{1}$. The trace of the magnetic field is unknown on $\Gamma_{f t, 1}$. Once it is determined from the solution in $\Omega_{1}$, it can be used as a $\mathrm{BC}$ for calculating the solution in $\Omega \backslash \Omega_{1}$, with all the precise characteristics of this exterior region (e.g., inductors and other regions). This task is however let to problem 2. For that, problem 1 gathers all the active parts of the exterior region inside the double layer defined by $\Gamma_{f t, 1}^{+}$and $\Gamma_{f t, 1}^{-}$, the inner and outer sides of $\Gamma_{f t, 1}$ with regard to $\Omega_{1}$ (Fig. 1, left). This allows the magnetic field to be zero in $\Omega \backslash \Omega_{1}$. One thus has

$$
\begin{gathered}
\left.\boldsymbol{n} \cdot \boldsymbol{b}_{1}\right|_{\Gamma_{f t, 1}^{+}}=0,\left.\quad \boldsymbol{n} \cdot \boldsymbol{b}_{1}\right|_{\Gamma_{f t, 1}^{-}}=0, \\
\boldsymbol{n} \times\left.\boldsymbol{h}_{1}\right|_{\Gamma_{f t, 1}^{+}}=\boldsymbol{j}_{s u, 1},\left.\quad \boldsymbol{n} \cdot \boldsymbol{h}_{1}\right|_{\Gamma_{f t, 1}^{-}}=0,
\end{gathered}
$$

or, for the discontinuities,

$$
\left[\boldsymbol{n} \cdot \boldsymbol{b}_{1}\right]_{\Gamma_{f t, 1}}=\boldsymbol{b}_{s u, 1}=0, \quad\left[\boldsymbol{n} \times \boldsymbol{h}_{1}\right]_{\Gamma_{f t, 1}}=\boldsymbol{j}_{s u, 1} \cdot
$$

Problem 2 must correct the solution 1 via particular ICs (1f-g). On the one hand,

$$
\left[\boldsymbol{n} \cdot \boldsymbol{b}_{2}\right]_{\Gamma_{f t, 2}}=\boldsymbol{b}_{s u, 2}=[\boldsymbol{n} \cdot \boldsymbol{b}]_{\Gamma_{f t, 2}}-\boldsymbol{b}_{s u, 1}=0
$$

due to the continuity of $\boldsymbol{n} \cdot \boldsymbol{b}$ in the complete solution (2) and the zero value of $\boldsymbol{b}_{s u, 1}$ via (9a). On the other hand,

$$
\left[\boldsymbol{n} \times \boldsymbol{h}_{2}\right]_{\Gamma_{f t, 2}}=\boldsymbol{j}_{s u, 2}=[\boldsymbol{n} \times \boldsymbol{h}]_{\Gamma_{f t, 2}}-\boldsymbol{j}_{s u, 1}=-\boldsymbol{n} \times\left.\boldsymbol{h}_{1}\right|_{\Gamma_{f t, 1}^{+}}
$$

due to the continuity of $\boldsymbol{n} \times \boldsymbol{h}$ in the complete solution (2) and relation (8a). Problem 2 has thus to extend the solution out of the flux tubes and to correct it in the tubes. IC (11) can be seen as a surface source acting on both sides of $\Gamma_{f t, 2}$. Note that $\Gamma_{f t, 2}$ is similar to $\Gamma_{f t, 1}$. They only differ at the discrete level due to their different meshes.

\section{B. From Surface to Volume Gaps}

The possible gaps in the flux tubes can be first approximated by surface (thin shell) FEs [4] in problem $p=1$, which simplifies the mesh of the whole structure. Another problem $p=2$ considers then the actual extension of the gaps with volume FEs. The associated studied domain $\Omega_{2}$ can be reduced to the neighborhood of the gap for improving the accuracy of the local solution, which allows the resulting fine mesh to be built only in a small domain.

In problem 1 , the gap $\Omega_{g, 1}$ of thickness $d_{1}$ is reduced to an average surface $\Gamma_{g, 1}$ situated halfway between its two main surfaces, with reluctivity $\left.\mu_{1}^{-1}\right|_{\Gamma_{g, 1}}=\mu_{0}^{-1}$. In problem 2 , the surface gap $\Gamma_{g, 2}=\Gamma_{g, 1}$ is then suppressed, via a modified reluctivity $\left.\mu_{2}^{-1}\right|_{\Gamma_{g, 2}}=0$, and simultaneously replaced by a volume gap, of reluctivity $\left.\mu_{2}^{-1}\right|_{\Omega_{g, 2}}=\mu_{0}^{-1}$. The two regiontype sources to be considered are respectively

$$
\left.\boldsymbol{h}_{s, 2}\right|_{\Gamma_{g, 2}}=-\mu_{1}^{-1} \boldsymbol{b}_{1},\left.\quad \boldsymbol{h}_{s, 2}\right|_{\Gamma_{g, 2}}=\left(\mu_{0}^{-1}-\mu_{1}^{-1}\right) \boldsymbol{b}_{1} \text {. }
$$

\section{FINITE ELEMENT WEAK FORMULATIONS}

\section{A. b-Conform Weak Formulation}

The canonical problem $p(1 \mathrm{a})-(\mathrm{g})$ is defined in $\Omega_{p}$ with the magnetic vector potential formulation [3], expressing the magnetic flux density $\boldsymbol{b}_{p}$ in $\Omega_{p}$ as the curl of a magnetic vector potential $\boldsymbol{a}_{p}$. The related $\boldsymbol{a}$-formulation is obtained from the weak form of the Ampère (1a), i.e. [3],

$$
\begin{aligned}
& \left(\mu_{p}^{-1} \operatorname{curl} \boldsymbol{a}_{p}, \operatorname{curl} \boldsymbol{a}^{\prime}\right)_{\Omega_{p}}-\left(\mu_{p}^{-1} \boldsymbol{b}_{s, p}, \operatorname{curl} \boldsymbol{a}^{\prime}\right)_{\Omega_{p}} \\
& \quad-\left(\boldsymbol{j}_{s, p}, \boldsymbol{a}^{\prime}\right)_{\Omega_{p}}+\left\langle\boldsymbol{n} \times \boldsymbol{h}_{s, p}, \boldsymbol{a}^{\prime}\right\rangle_{\Gamma_{h, p}}+\left\langle\boldsymbol{n} \times \boldsymbol{h}_{p}, \boldsymbol{a}^{\prime}\right\rangle_{\Gamma_{b, p}}
\end{aligned}
$$




$$
+\left\langle\left[\boldsymbol{n} \times \boldsymbol{h}_{p}\right]_{\gamma_{p}}, \boldsymbol{a}^{\prime}\right\rangle_{\gamma_{p}}=0, \quad \forall \boldsymbol{a}^{\prime} \in F_{p}^{1}\left(\Omega_{p}\right),
$$

where $F_{p}^{1}\left(\Omega_{p}\right)$ is a gauged curl-conform function space defined on $\Omega_{p}$ and containing the basis functions for $\boldsymbol{a}$ as well as for the test function $\boldsymbol{a}^{\prime}$ (at the discrete level, this space is defined by edge finite elements); $(\cdot, \cdot)_{\Omega}$ and $\langle\cdot, \cdot\rangle_{\Gamma}$ respectively denote a volume integral in $\Omega$ and a surface integral on $\Gamma$ of the product of their vector field arguments. The surface integral term on $\Gamma_{h, p}$ accounts for natural BCs of type (1d), usually with $\boldsymbol{n} \times\left.\boldsymbol{h}_{s, p}\right|_{\Gamma_{h, p}}=0$. The term on the surface $\Gamma_{b, p}$ with essential BCs on $\boldsymbol{n} \cdot \boldsymbol{b}_{p}$ is usually omitted because it does not locally contribute to (13). It will be shown to be the key for the post-processing of a solution, a part of which being $\boldsymbol{n} \times\left.\boldsymbol{h}_{p}\right|_{\Gamma_{b, p}}$.

A major consequence of the $\boldsymbol{b}$-conform formulation used is that ICs $(1 \mathrm{~g})$ and (1f) are to be defined respectively in strong and weak senses, i.e. in $F_{p}^{1}\left(\Omega_{p}\right)$ and in a surface integral term.

\section{B. From Ideal to Real Flux Tubes}

For the ideal flux tubes $\Gamma_{f t, 1}$ of problem 1, BC (7a) leads to an essential BC on the primary unknown $\boldsymbol{a}_{1}$ that can be expressed in general (in 3-D) via the definition of a surface scalar potential $u_{1}$ (multi-valued because a net magnetic flux flows in $\Omega_{f t, 1}$ ) [3], i.e.,

$$
\left.\boldsymbol{n} \cdot \operatorname{curl} \boldsymbol{a}_{1}\right|_{\Gamma_{f t, 1}}=0 \Leftrightarrow \boldsymbol{n} \times\left.\boldsymbol{a}_{1}\right|_{\Gamma_{f t, 1}}=\boldsymbol{n} \times\left.\operatorname{grad} u_{1}\right|_{\Gamma_{f t, 1}}
$$

or via a floating $\boldsymbol{a}_{1}$ in 2-D (a constant for the perpendicular component of $\boldsymbol{a}_{1}$ on each non-connected part of $\left.\Gamma_{f t, 1}\right)$.

Formulation $p=1$ is obtained from (13) with $\boldsymbol{b}_{s, 1}=0$, $\boldsymbol{j}_{s, 1}=0, \boldsymbol{n} \times\left.\boldsymbol{h}_{s, 1}\right|_{\Gamma_{h, 1}}=0, \Gamma_{f t, 1} \subset \Gamma_{b, 1}$ and $\gamma_{1}=\varnothing$.

The surface integral term $\left\langle\boldsymbol{n} \times \boldsymbol{h}_{1}, \boldsymbol{a}^{\prime}\right\rangle_{\Gamma_{f t, 1}}$ is non-zero only for the function grad $u^{\prime}$ (from (14)), the value of which is then the MMF $F_{1}$ associated with a flux tube (this can be demonstrated from the general procedure developed in [3]). It is zero for all the other local test functions (at the discrete level, for any edge not belonging to $\Gamma_{f t, 1}$ ). This way, the magnetic circuit relation can be expressed for each flux tube $\Omega_{f t, 1}$, to relate fluxes and MMFs.

The correction formulation $p=2$ is then obtained from (13) with $\boldsymbol{b}_{s, 2}=0, \boldsymbol{n} \times\left.\boldsymbol{h}_{s, 2}\right|_{\Gamma_{h, 2}}=0$ and $\gamma_{2}=\Gamma_{f t, 2}$. The source $j_{s, 2}$ is now defined in the inductor portions added to $\Omega_{2}$, in place of the idealized inductors. IC (10) is strongly expressed via the continuity of the vector potential $\boldsymbol{a}_{2}$ through $\Gamma_{f t, 2}$. IC (11) can rather only act in a weak sense via the surface integral term related to $\gamma_{2}=\Gamma_{f t, 2}$ in (13). Indeed, the involved surface source $\boldsymbol{n} \times \boldsymbol{h}_{1}$ is not known in a strong sense on $\Gamma_{f t, 2}$, but rather in a weak sense. One has

$$
\begin{aligned}
\left\langle\left[\boldsymbol{n} \times h_{2}\right]_{\Gamma_{f t, 2}}, \boldsymbol{a}^{\prime}\right\rangle_{\Gamma_{f t, 2}} & =\left\langle-\boldsymbol{n} \times \boldsymbol{h}_{1}, \boldsymbol{a}^{\prime}\right\rangle_{\Gamma_{f t, 2}^{+}} \\
& =\left\langle-\boldsymbol{n} \times \boldsymbol{h}_{1}, \boldsymbol{a}^{\prime}\right\rangle_{\Gamma_{f t, 1}^{+}} \\
& =\left(\mu_{1}^{-1} \operatorname{curl} \boldsymbol{a}_{1}, \operatorname{curl} \boldsymbol{a}^{\prime}\right)_{\Omega_{f t, 1}=\Omega_{f t, 2}}
\end{aligned}
$$

This way, the surface integral term related to $\Gamma_{f t, 2}$ in (13) is calculated from a volume integral coming from the first problem. Its consideration via a volume integral, limited at the discrete level to one single layer of FEs touching the boundary, is the natural way to average it as a weak quantity.

At the discrete level, the source quantity $\boldsymbol{a}_{1}$ in (15) has to be expressed in the mesh of problem 2, while it is initially given in

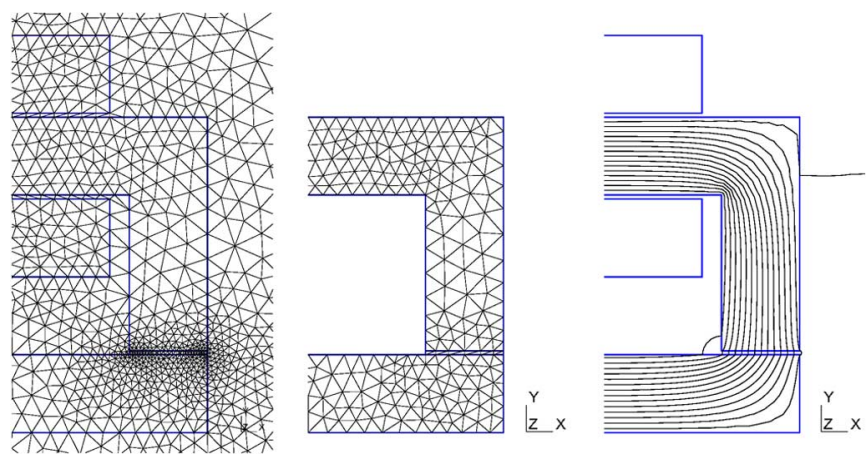

Fig. 2. Meshes (half portions) of the whole studied domain (left) and the ideal flux tube (middle); field lines of the complete solution (b, right).
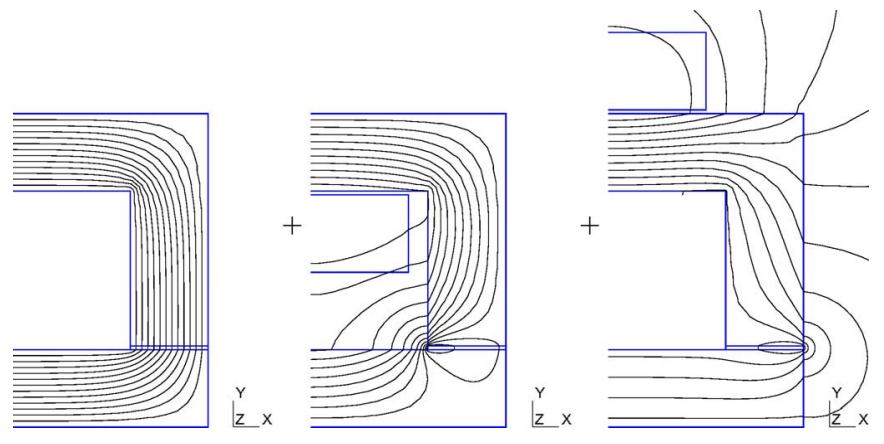

Fig. 3. Field lines in the ideal flux tube $\left(\boldsymbol{b}_{1}\right.$, left $)$ and in the perturbation problems with the inner $\left(\boldsymbol{b}_{2}\right.$, middle $)$ and outer $\left(\boldsymbol{b}_{3}\right.$, right $)$ leakage fluxes.

the mesh of problem 1 . This can be done via a projection method [2] of its curl limited to the layer of FEs touching $\Gamma_{f t, 2}$.

\section{From Surface to Volume Gaps}

For problem 1, the surface representation $\Gamma_{g, 1}$ of the gap $\Omega_{g, 1}$ amounts to express the first term of (13) in this region as

$\left(\mu_{1}^{-1} \operatorname{curl} \boldsymbol{a}_{1}, \operatorname{curl} \boldsymbol{a}^{\prime}\right)_{\Omega_{g, 1}}=\left\langle d_{1} \mu_{1}^{-1} \operatorname{curl} \boldsymbol{a}_{1}, \operatorname{curl} \boldsymbol{a}^{\prime}\right\rangle_{\Gamma_{g, 1}}$

For problem 2, the surface gap is suppressed via the source (12a) in

$$
\left(\boldsymbol{h}_{s, 2}, \operatorname{curl} \boldsymbol{a}^{\prime}\right)_{\Omega_{g, 1}}=\left\langle-d_{1} \mu_{1}^{-1} \operatorname{curl} \boldsymbol{a}_{1}, \operatorname{curl} \boldsymbol{a}^{\prime}\right\rangle_{\Gamma_{g, 2}=\Gamma_{g, 1}}
$$

and simultaneously replaced by a volume gap $\Omega_{g, 2}$ via

$$
\left(\mu_{2}^{-1} \operatorname{curl} \boldsymbol{a}_{2}, \operatorname{curl} \boldsymbol{a}^{\prime}\right)_{\Omega_{g, 2}}+\left(\boldsymbol{h}_{s, 2}, \operatorname{curl} \boldsymbol{a}^{\prime}\right)_{\Omega_{g, 2}}
$$

with $\left.\mu_{2}^{-1}\right|_{\Omega_{g, 2}}=\mu_{0}^{-1}$ and source $\left.h_{s, 2}\right|_{\Omega_{g, 2}}$ given by (12b).

These terms can be used as well for any variation of the permeability of a thin shell. A variation of the thickness of the gap from $d_{1}$ to $d_{2}$ can be simply considered via the source

$$
\left(\boldsymbol{h}_{s, 2}, \operatorname{curl} \boldsymbol{a}^{\prime}\right)_{\Omega_{g, 2}}=\left\langle\left(d_{2}-d_{1}\right) \mu_{1}^{-1} \operatorname{curl} \boldsymbol{a}_{1}, \operatorname{curl} \boldsymbol{a}^{\prime}\right\rangle_{\Gamma_{g, 2}=\Gamma_{g, 1}} \text {. }
$$

At the discrete level, the source quantity $\boldsymbol{a}_{1}$ in (16)-(18) needs to be projected from the mesh of problem 1 to the mesh of problem 2, only in $\Gamma_{g, 2}$ and $\Omega_{g, 2}$.

\section{APPLiCATION EXAMPLE}

An electromagnet is considered to test and illustrate the method. It consists of a U-shape core surrounded by a stranded inductor and separated from an I-shape core via two air gaps (Fig. 2). An approximate solution $p=1$ is first calculated in 

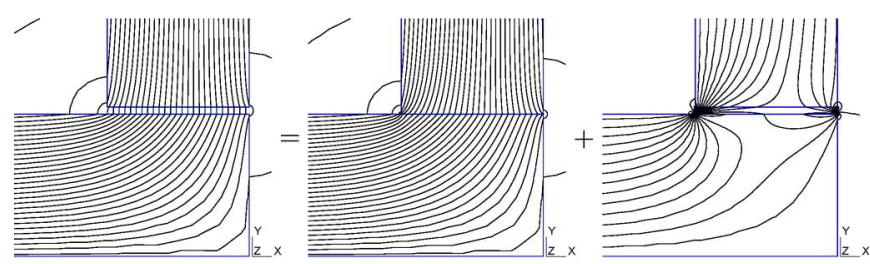

Fig. 4. Field lines of the complete solution $(\boldsymbol{b}$, left $)$, of the initial problem with surface gap $\left(\boldsymbol{b}_{1}\right.$, middle $)$ and its correction to a volume gap $\left(\boldsymbol{b}_{2}\right.$, right $)$.
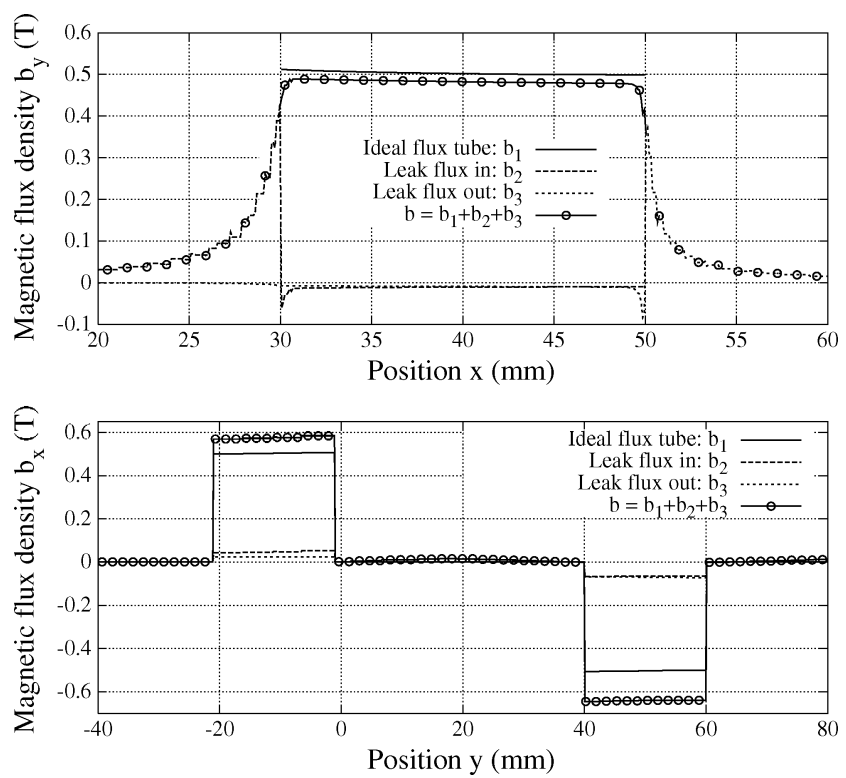

Fig. 5. Magnetic flux density along the top surface of the I-core (entering the air gap; top) and through the horizontal legs of the electromagnet (bottom) for the ideal flux tube $\left(\boldsymbol{b}_{1}\right)$ and the inner $\left(\boldsymbol{b}_{2}\right)$ and outer $\left(\boldsymbol{b}_{3}\right)$ leakage fluxes; their addition gives the complete solution $(\boldsymbol{b})$.

an idealized flux tube (Fig. 3, left), with a fixed magnetomotive force as excitation and a coarse mesh of the tube (Fig. 2, middle). This solution serves then as a source for a perturbation problem $p=2$ allowing leakage flux in the inner region of the core (Fig. 3, middle), followed by another problem $p=3$ allowing leakage flux in the outer region (Fig. 3, right). Each of these problems calculates the actual flux distribution in the related inductor portion and in the vicinity of the gaps, with its own adapted mesh. They also correct the flux density in the cores. Another sequence of problems considers a solution 1 for the surface gaps (thin shell model), followed by its correction for the volume gaps (Fig. 4), in a locally refined mesh.

The magnetic flux density along one gap and through core portions is shown for the two sequences of problems in Figs. 5 and 6 . The thin shell model significantly overestimates the flux density near the gap borders (mainly in a portion comparable to the gap thickness; equal to $1 \mathrm{~mm}$ for a gap length of $20 \mathrm{~mm}$ ), which is perfectly corrected by problem 2 . For both sequences, the error is not only limited to the gap region but also to the flux in the core, as shown in Figs. 5 and 6 (bottom). Each corrected solution has been checked to be in perfect accordance with the one-step complete FE solution.
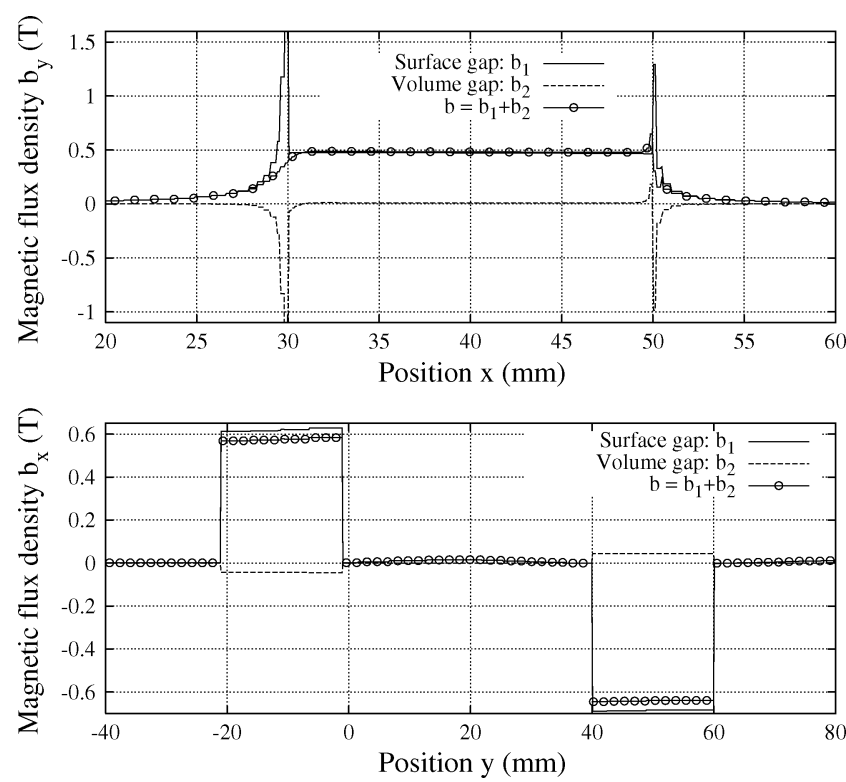

Fig. 6. Magnetic flux density along the top surface of the I-core (entering the air gap; top) and through the horizontal legs of the electromagnet (bottom) for the surface gap $\left(\boldsymbol{b}_{1}\right)$ and its correction to a volume gap $\left(\boldsymbol{b}_{2}\right)$.

\section{CONCLUSION}

The developed perturbation FE method splits magnetic circuit analyses into problems of lower complexity regarding meshing operations and computational aspects. This allows a natural progression from simple to elaborate models, while quantifying the gain given by each model refinement to justify its utility. Approximate problems with ideal flux tubes and/or thin shell models for gaps are accurately corrected. Additional refinements towards eddy current or 3-D effects are possible extensions. All the constraints involved in the subproblems have been carefully defined in the resulting FE formulations, respecting their inherent strong and weak natures. As a result, an efficient and accurate computation of local fields and global quantities (e.g., flux, MMF, reluctance) is obtained.

\section{ACKNOWLEDGMENT}

This work was supported in part by the Belgian Science Policy (IAP P6/21), the Belgian French Community (Research Concerted Action ARC 03/08-298), and the Walloon Region.

\section{REFERENCES}

[1] Z. Badics, Y. Matsumoto, K. Aoki, F. Nakayasu, M. Uesaka, and K. Miya, "An effective 3-D finite element scheme for computing electromagnetic field distortions due to defects in eddy-current nondestructive evaluation," IEEE Trans. Magn., vol. 33, no. 2, pp. 1012-1020, 1997.

[2] P. Dular, R. V. Sabariego, J. Gyselinck, and L. Krähenbühl, "Sub-domain finite element method for efficiently considering strong skin and proximity effects," COMPEL, vol. 26, no. 4, pp. 974-985, 2007.

[3] P. Dular, J. Gyselinck, T. Henneron, and F. Piriou, "Dual finite element formulations for lumped reluctances coupling," IEEE Trans. Magn., vol. 41, no. 5, pp. 1396-1399, 2005.

[4] C. Guerin, G. Tanneau, G. Meunier, X. Brunotte, and J. B. Albertini, "Three dimensional magnetostatic finite elements for gaps and iron shells using magnetic scalar potentials," IEEE Trans. Magn., vol. 30, no. 5, pp. 2885-2888, 1994. 